\title{
Cooperative Communication, Localization, Sensing and Control for Autonomous Robotic Networks
}

\author{
Siwei Zhang, Emanuel Staudinger, Robert Pöhlmann and Armin Dammann \\ Institute of Communications and Navigation, German Aerospace Center (DLR), Oberpfaffenhofen, Germany \\ Email: firstname.lastname@DLR.de
}

\begin{abstract}
Networks composed of a myriad of autonomous robots have attracted increasing attention in recent years due to their enormous capability expansion from single robot systems. In these networks, robots benefit from the collaboration with each other to enhance their situation awareness for autonomous operation. For example, in an extraterrestrial exploration mission, a robotic swarm can collaboratively utilize the inter-robot communication system to propagate information, synchronize itself, and navigate to achieve mission objectives like joint environmental sensing. In addition, each robot can decide and control its own trajectory, so that the aforementioned tasks are accomplished in a globally efficient manner. In this paper, we propose multi-agent control strategies for autonomous robotic networks, which adapt the mission demands on cooperative communication, localization and sensing. We also discuss three space exploration examples with different mission demands, which lead to distinct network formations. These three missions will be conceptually demonstrated in a space analog mission on the volcano Mount Etna in June 2022.
\end{abstract}

\section{INTRODUCTION}

A swarm of autonomous robots [1], analog to a biological swarm in nature [2], can rapidly explore a vast area on earth or in space, make simultaneous observations at different locations, and avoid a single point of failure. Therefore, it is a promising concept and a paradigm shift in exploration of human inaccessible area, e.g. search-and-rescue [3], environmental monitoring [4] and future space missions [1], [5]. A major challenge of such an exploration is communication and navigation, since external infrastructures like cellular networks or global navigation satellite systems (GNSSs) are often absent in a human inaccessible area. In this case, the network composed of a myriad of robots can additionally provide communication and localization service in the area as a temporal infrastructure, next to its exploration tasks like collaborative environment sensing and target tracking. Regarding autonomy, every robot is free to choose its trajectory, jointly considering the requirements of cooperative communication, localization and exploration. It is a multi-agent, multi-objective optimization problem, which often needs to be solved in-situ due to the unpredictable environmental situations. In [6] we have proposed an autonomous swarm navigation framework, where a swarm optimizes its formation to improve localization, while achieving mission objectives.

In this paper, we have a joint look at the communication, localization, sensing and control aspects in an exploration mission, which is essential for design such an autonomous robotic network [7], [8]. Depending on the overall mission objectives,

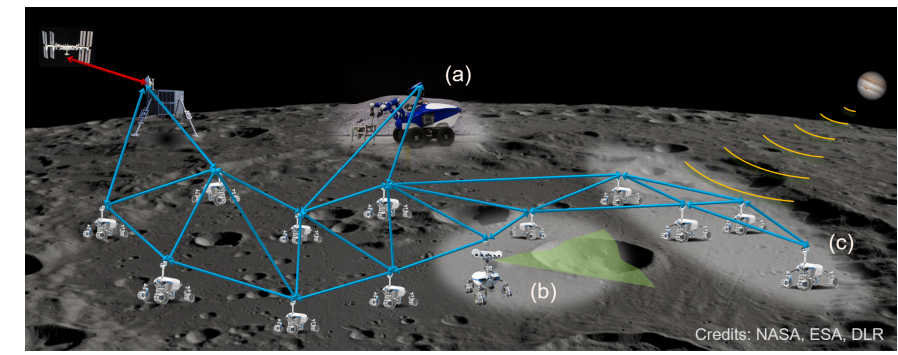

Figure 1: Concepts of three lunar swarm missions: (a) remote rover manipulation, (b) geological exploration and (c) LOFAR.

the trade-off between communication and localization determines the favorable swarm formations. We investigate this trade-off with three representative swarm exploration missions, namely remote rover manipulation, geological exploration and low frequency array (LOFAR). We extend the autonomous swarm navigation framework from [6] with cooperative communication. It allows the swarm to adapt its formation according to the communication-localization trade-off, ergo the mission objectives. Both simulations and experiments have been conducted to prove the concept of autonomous swarm navigation, which will be demonstrated in the space-analog mission planned on volcano Mount Etna, Italy, in 2022 [9].

\section{LUNAR SWARM EXPLORATION CONCEPTS}

We study three lunar swarm exploration concepts, as shown in Figure 1. A swarm of rovers move from the mission base to operation areas, while maintaining localizability and communication.

In the first mission, one rover at area (a) is steered remotely by astronauts from an orbiter. For this mission, the localization requirement is relatively low. However, a reliable communication between the rover and the orbiter needs to be guaranteed for real-time video and sensor data streaming.

In the second mission at area (b), two rovers are mapping the environment for geological exploration. In order to collectively reconstruct the environmental map, large volume of sensor data, together with its precise collecting location, need to be frequently exchanged. In this mission, both communication and localization are demanding.

In the third mission, four rovers at area (c) form a LOFAR for radio astronomy. As an example, they receive the radio bursts of Jupiter and determine the direction of Jupiter with respect to (w.r.t.) the swarm. In this case, the location 
estimates of the rovers have to be sufficiently precise, while communication is less demanding.

In these lunar exploration concepts, the communicationlocalization trade-off varies according to the application. Next, we look into the design of the swarm control, optimizing the swarm formation for cooperative localization, communication and mission objectives like target tracking, respectively.

\section{Multi-Agent Control}

Let us consider a swarm of robots with their positions at time $t$ collectively denoted as $\mathbf{p}_{\mathcal{A}}^{(t)}$. Robots' positions can be manipulated with a control command $\mathbf{u}_{\mathcal{A}}^{(t)}$ into $\mathbf{p}_{\mathcal{A}}^{(t+1)}$ as

$$
\mathbf{p}_{\mathcal{A}}^{(t+1)}=f\left(\mathbf{p}_{\mathcal{A}}^{(t)}, \mathbf{u}_{\mathcal{A}}^{(t)}, \boldsymbol{\epsilon}_{\mathcal{A}}^{(t)}\right),
$$

where $\epsilon_{\mathcal{A}}^{(t)}$ denotes the controller noise. As an example of sensing applications, we consider tracking a target at position $\mathbf{p}_{\mathrm{t}}^{(t)}$. A robot in the network is referred to as 'leader' with position $\mathbf{p}_{1}^{(t)}$, which aims at following the target. Another static robot at position $\mathbf{p}_{\mathrm{s}}^{(t)}$ close to the mission base is referred to as 'sink'. As an example of communication requirements, we consider nonspecific sensor data about the target transmitted from the leader to the sink over the network. Communication and distance measurements between entities can be conducted if the entities are within their respective communication range. Three static entities close to the mission base serve as anchors with known positions. Both positions of the robots and targets need to be estimated with distance measurements in the network $\mathbf{z}^{(t)}$. The overall executed control command $\tilde{\mathbf{u}}_{\mathcal{A}}^{(t)}$ can be derived, for example with a linear combination of the controls for localization $\mathbf{u}_{\mathrm{L}}^{(t)}$, target tracking $\mathbf{u}_{\mathrm{T}}^{(t)}$ and communication $\mathbf{u}_{\mathrm{C}}^{(t)}$. The individual control commands are introduced next.

\section{A. Cooperative Localization}

For cooperative localization, we aim at finding an optimized control command $\mathbf{u}_{\mathrm{P}}^{(t)}$, so that the predicted measurements $\tilde{\mathbf{z}}^{(t+1)}$ provide the richest information for estimating the new positions $\mathbf{p}_{\mathcal{A}}^{(t+1)}$, i.e.

$$
\mathbf{u}_{\mathrm{P}}^{(t)}=\underset{\mathbf{u}_{\mathcal{A}}^{(t)}}{\arg \min } \mathbb{E}\left[\left\|\mathbf{p}_{\mathcal{A}}^{(t+1)}-\hat{\mathbf{p}}_{\mathcal{A}}^{(t+1)}\right\|^{2}\right] .
$$

We employ the information seeking control proposed in [6] to solve (2). The estimation uncertainty is inferred with the Cramér-Rao bound (CRB), i.e.

$$
\mathrm{CRB}\left[\mathbf{p}_{\mathcal{A}}^{(t+1)}\right] \preccurlyeq \mathbb{E}\left[\left\|\mathbf{p}_{\mathcal{A}}^{(t+1)}-\hat{\mathbf{p}}_{\mathcal{A}}^{(t+1)}\right\|^{2}\right] .
$$

The optimized control command is proportional to the negative gradient of the CRB, i.e.

$$
\mathbf{u}_{\mathrm{L}}^{(t)} \propto-\nabla_{\mathbf{u}_{\mathcal{A}}^{(t)}} \mathrm{CRB}\left[\mathbf{p}_{\mathcal{A}}^{(t+1)}\right]
$$

\section{B. Cooperative Target Tracking}

The objective of cooperative target tracking is twofold. Firstly, all the robots compose a sensor array whose formation is favorable for estimating the position of the target, hence minimizing the position CRB of the target:

$$
\mathbf{u}_{\mathrm{T}}^{(t)} \propto-\nabla_{\mathbf{u}_{\mathcal{A}}^{(t)}} \operatorname{CRB}\left[\mathbf{p}_{\mathrm{t}}^{(t+1)}\right]
$$

Secondly, the leader moves towards the target with an additional control command

$$
\mathbf{u}_{\mathrm{L}}^{(t)}=\underset{\mathbf{u}_{\mathcal{A}}^{(t)}}{\arg \min }\left\|\mathbf{p}_{1}^{(t+1)}-\mathbf{p}_{\mathrm{t}}^{(t+1)}\right\| .
$$

\section{Cooperative Communication}

The robotic network can be considered as an undirected graph with robots as the vertices. If two vertices $i$ and $j$ are within the communication range of each other, they are connected with an edge $l_{i j}$. The maximum throughput on this edge is expressed with the Shannon capacity:

$$
C_{i j}=B \log _{2}\left(1+\mathrm{SNR}_{i j}\right),
$$

where $B$ is the bandwidth and $\mathrm{SNR}_{i j}$ is the signal to noise ratio (SNR) of the edge which is proportional to the Euclidean distance $d_{i j}$ between vertices $i$ and $j$. We assume a decodeand-forward relaying scheme for all the robots. The control strategy for communication can be formulated as finding a suitable route $\mathcal{L}=\left\{\cdots l_{i j}, \cdots\right\}$ from the leader to the sink, and determining a control command $\mathbf{u}_{\mathrm{C}}^{(t)}$ that minimizes all edge distances in the route, i.e.

$$
\mathbf{u}_{\mathrm{C}}^{(t)}=\underset{\mathbf{u}_{\mathcal{A}}^{(t)}}{\arg \min }\left\{d_{i j}: \forall l_{i j} \in \mathcal{L}\right\} .
$$

A suitable route can either be a single path connecting the leader and the sink, or include multiple paths. Multiple strategies can be exploited for finding a suitable route.

1) Pre-Defined Path: A naive strategy is to pre-define a fixed route from the leader to the sink. For example, half of the robots in the network are chosen as relays.

2) Shortest Path: The shortest path is the one with minimal number of relays in between. The Dijkstra's algorithm [10] can be applied to find the shortest path. This strategy is suitable when the communication requirement is less demanding like in the LOFAR mission.

3) Widest Path: The widest path is a single path which has the highest throughput. Therefore it is also referred to as the maximum capacity problem [11]. For the decode-and-forward relaying model, the total throughput equals the minimum edge capacity. Hence finding the widest path is equivalent to finding a path whose longest edge is the shortest among all possible paths. It can be achieved with a modification of the Dijkstra's algorithm. A route with widest path is suitable when the communication requirement is as demanding as other requirements like in the geological exploration mission.

4) Maximum Flow: The maximum flow strategy exploits the whole network for communication with high priority. It maximizes the total throughput allowing multiple paths. The Boykov-Kolmogorov algorithm [12] can be utilized to calculate the route with maximum flow. This strategy is suitable for 


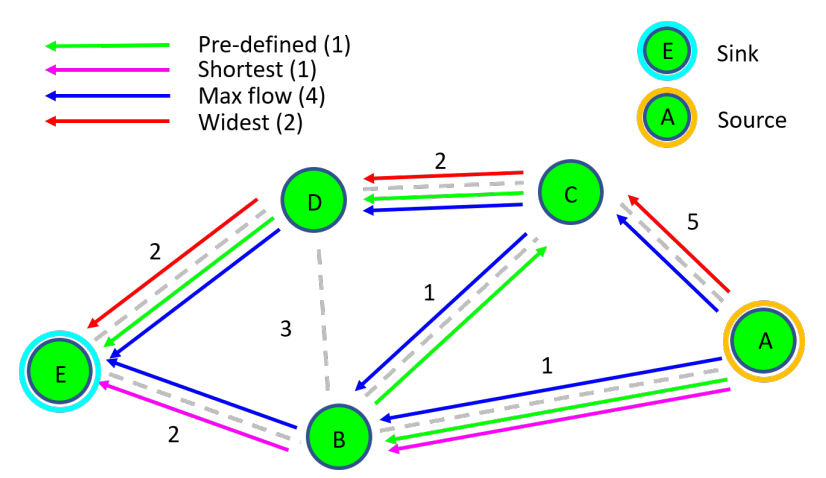

Figure 2: Different routing strategies for a toy scenario.

demanding communication requirements like the rover remote manipulation mission.

The above-mentioned routing strategies are illustrated in Figure 2. The edge capacity is labelled at the individual edge. The total route capacities are shown in the legend.

\section{RESUlts}

\section{A. Simulations}

We conduct swarm control simulations taking five different strategies for cooperative communication, namely no communication requirement considered (shortest path for calculating throughput), pre-defined path, shortest path, widest path and maximum flow. Three simulation snapshots are shown in Figure 3. A swarm of 40 robots (green markers) depart from three anchors (blue markers) to track a target (magenta markers) beyond the communication range of the anchors (magenta dashed lines). The leader and sink are labelled with orange and cyan circles, respectively. Edges with distance measurements, communication and communication with full capacity are indicated with gray, blue and red lines, respectively. In all three scenarios, robots build a rigid bridge connecting anchors, swarm and the target, so that the swarm and target are localizable. In the case of no communication requirement, the leader reaches the target. In the case of widest path, the relays form almost a straight line to have a high total path capacity. In the case of maximum flow, the route contains multiple paths with high relay concentration. In this way the total route capacity is maximized. A four dimensional performance comparison of different swarm control strategies, normalized to the respective maximum value, is shown in Figure 4. Maximum flow based control guarantees the highest throughput at the cost of having the largest number of hops. No communication requirement or shortest path based control experience a large communication latency, but consume less hops and are more efficient in localization and target following. Widest path based control is well balanced in all four metrics and outperforms the pre-defined path approach. As a conclusion, the proposed strategies can be used in swarm control, according to the desired communication-localization trade-off.

\section{B. Experiments}

Within the project Autonomous Robotic Networks to Help Modern Societies (ARCHES) we will demonstrate robotic
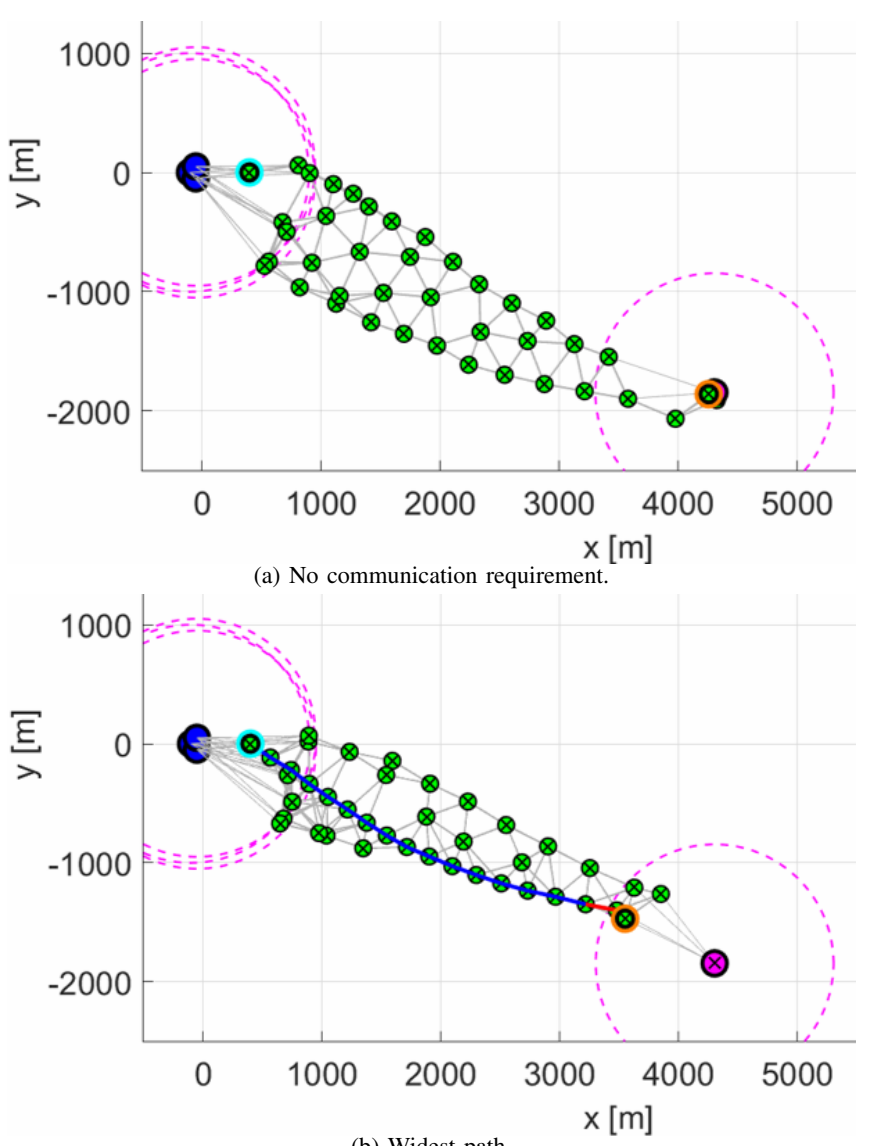

(b) Widest path

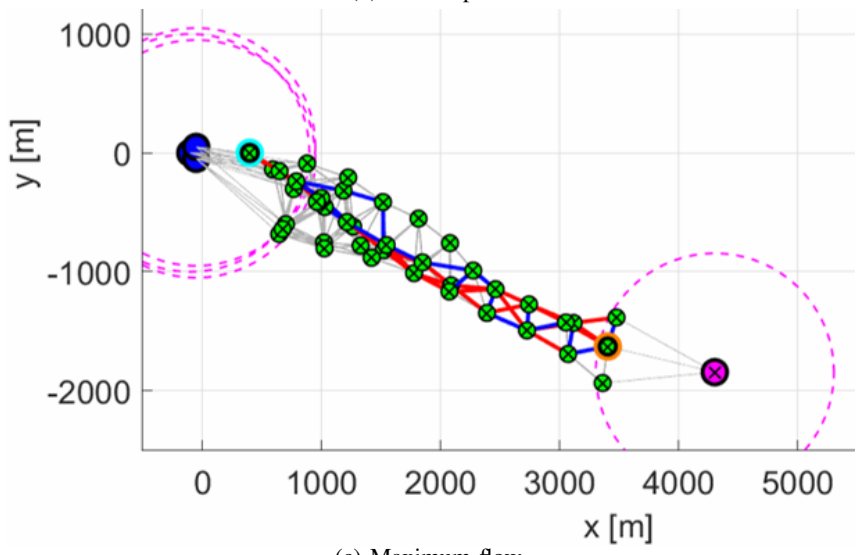

(c) Maximum flow.

Figure 3: Swarm formation snapshots with different cooperative communication strategies.

exploration technologies in a lunar analog environment on Mount Etna, Italy, in 2022 [9]. The demonstration mission consists of three scenarios that are closely related to the three conceptual missions introduced in Section II. The first two scenarios examine technical and operational aspects of geological in-situ analysis and sample return, with rovers either manipulated remotely by an ESA astronaut, or operating autonomously. In these scenarios, static boxes or rovers are deployed at preferable positions as relays to guarantee reliable communication. The third scenario demonstrates the autonomous deployment of a LOFAR, which is illustrated in 


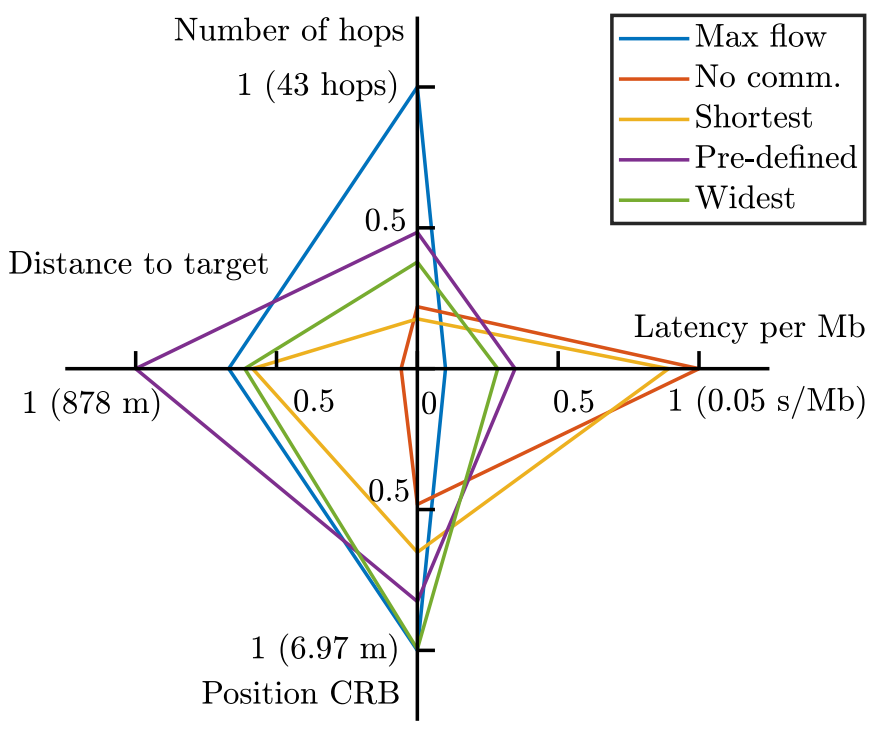

Figure 4: Performance comparison of different swarm control strategies for communication.

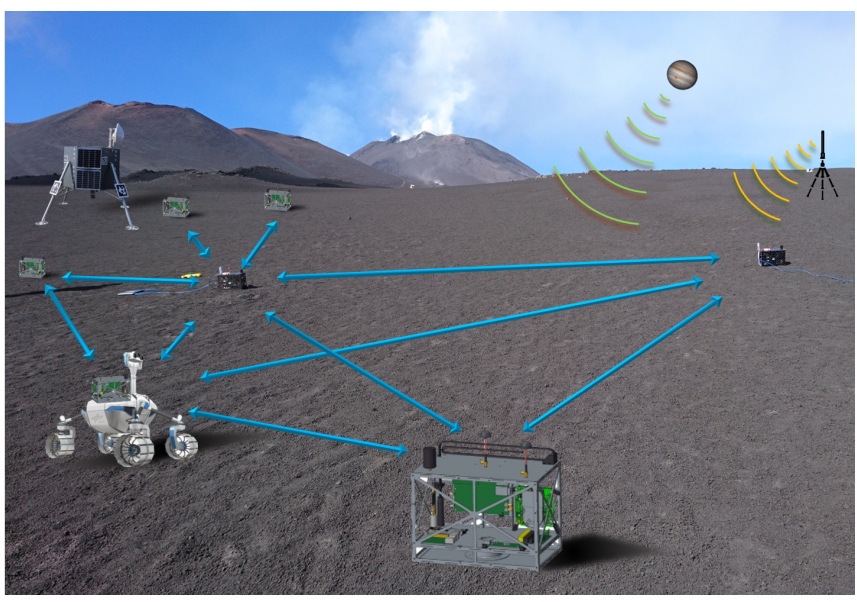

Figure 5: Space-analog LOFAR mission in 2022.

Figure 5. LOFAR payload boxes are placed by a lightweight rover and precisely synchronized and localized with our swarm navigation system [8]. Low frequency radio signals emitted either from space or by an artificial transmitter are detected by this array. As we discussed in Section II, in this scenario the most demanding requirement is localization.

As a preparation to this space analog mission, we have conducted a LOFAR experiment with our developed swarm navigation platform [7] at the German Aerospace Center (DLR) in March 2021. The experimental setup with four LOFAR boxes and two low frequency transmitters is shown in Figure 6. Software defined radio is used to generate and receive radio signals. Among LOFAR boxes and anchors, radio signals with a carrier frequency around $5.5 \mathrm{GHz}$ and a bandwidth of 25 $\mathrm{MHz}$ are transmitted for time of flight ( $\mathrm{ToF})$ based distance measurement. From the low frequency transmitters, sine-waves with a carrier frequency around $20 \mathrm{MHz}$ are transmitted. A real-time decentralized particle filter [13] is implemented for each LOFAR box for swarm cooperative localization. The

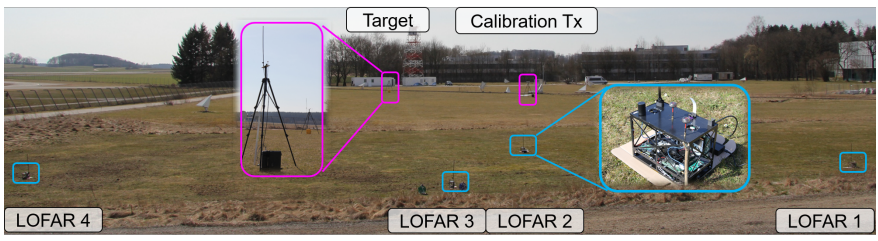

Figure 6: Swarm self localization experiment as preparation of LOFAR mission.

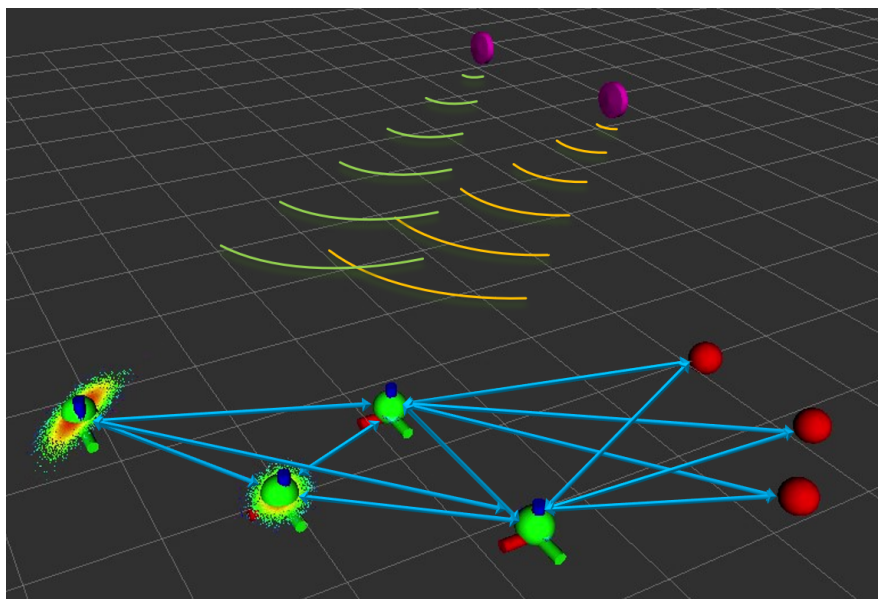

Figure 7: Experimental results for swarm localization.

localization result can be seen in Figure 7. The ground-truth positions of three anchors, four LOFAR boxes and two low frequency transmitters are measured with the GNSS-real-time kinematic (RTK) systems, illustrated with red markers, axes, and magenta markers. The point estimates of the LOFAR boxes' positions are indicated with green markers, surrounded by particles. Due to the curvature of the ground and low box's elevation, LOFAR boxes three and four cannot obtain distance measurements from any anchors. However, they are still able to estimate their positions through cooperation with LOFAR boxes one and two, with larger position uncertainty. Sub-meter position uncertainty can be obtained even for LOFAR boxes that do not directly connected with anchors. This accuracy is at least an order of magnitude smaller than the wavelength of the low frequency signals $(15 \mathrm{~m})$, ergo sufficient for LOFAR mission [8]. The LOFAR signals are detected by all LOFAR boxes coherently. As an ongoing work, we are analysing the performance of the direction of arrival (DoA) estimation of the low frequency transmitters.

\section{CONClusion}

In this paper, we proposed a swarm control framework which jointly considers the mission requirements for communication, localization and sensing. Multiple strategies can be chosen according to the desired communication-localization trade-off for different space exploration missions. Simulations verify the proposed swarm control framework, while experiments prove the concept of swarm cooperative localization in a LOFAR mission which will be demonstrated in a space analog mission on Mount Etna in 2022. 


\section{ACKNOWLEDGMENT}

Part of the presented research has been supported by the Helmholtz Association project ARCHES (contract number ZT0033). The authors would like to thank Kimon Cokona for his support.

\section{REFERENCES}

[1] E. Vassev, R. Sterritt, C. Rouff, and M. Hinchey, "Swarm technology at NASA: Building resilient systems," IEEE IT Prof., vol. 14, no. 2, pp. 36-42, Mar. 2012.

[2] M. Ballerini et al., "Interaction ruling animal collective behavior depends on topological rather than metric distance: Evidence from a field study," Proceedings of the National Academy of Sciences, vol. 105, no. 4, pp. 1232-1237, 2008.

[3] M. Bernard, K. Kondak, I. Maza, and A. Ollero, "Autonomous transportation and deployment with aerial robots for search and rescue missions," J. Field Robot., vol. 28, no. 6, pp. 914-931, 2011.

[4] M. Dunbabin and L. Marques, "Robots for environmental monitoring: Significant advancements and applications," IEEE Robot. Autom. Mag., vol. 19, no. 1, pp. 24-39, Mar. 2012.

[5] A. Seeni, B. Schfer, and G. Hirzinger, "Robot mobility systems for planetary surface exploration - state-of-the-art and future outlook: A literature survey," in Aerospace Technologies Advancements, T. T., Ed. London: InTech, Jan. 2010, pp. 189-208.

[6] S. Zhang, R. Pöhlmann, T. Wiedemann, A. Dammann, H. Wymeersch, and P. A. Hoeher, "Self-aware swarm navigation in autonomous exploration missions," Proc. IEEE, vol. 108, no. 7, pp. 1168-1195, 2020.

[7] S. Zhang, R. Pöhlmann, E. Staudinger, and A. Dammann, "Assembling a swarm navigation system: Communication, localization, sensing and control," in 2021 IEEE 18th Annual Consumer Communications Networking Conference (CCNC), 2021, pp. 1-9.

[8] E. Staudinger, S. Zhang, R. Poehlmann, and A. Dammann, "The role of time in a robotic swarm: A joint view on communications, localization, and sensing," IEEE Commun. Mag., vol. 59, no. 2, pp. 98-104, 2021.

[9] M. J. Schuster et al., "The ARCHES space-analogue demonstration mission: Towards heterogeneous teams of autonomous robots for collaborative scientific sampling in planetary exploration," IEEE Robotics and Automation Letters, pp. 1-1, 2020.

[10] E. W. Dijkstra, "A note on two problems in connexion with graphs," Numer. Math., vol. 1, no. 1, p. 269-271, Dec. 1959. [Online]. Available: https://doi.org/10.1007/BF01386390

[11] M. Pollack, "The maximum capacity through a network," Operations Research, vol. 8, no. 5, pp. 733-736, 1960. [Online]. Available: http://www.jstor.org/stable/167387

[12] Y. Boykov and V. Kolmogorov, "An experimental comparison of mincut/max- flow algorithms for energy minimization in vision," IEEE Trans. Pattern Anal. Mach. Intell., vol. 26, no. 9, pp. 1124-1137, 2004.

[13] S. Zhang, E. Staudinger, T. Jost, W. Wang, C. Gentner, A. Dammann, H. Wymeersch, and P. A. Hoeher, "Distributed direct localization suitable for dense networks," IEEE Trans. Aerosp. Electron. Syst., vol. 56, no. 2, pp. 1209-1227, 2020. 